\title{
Higher powered magnification improved endodontic surgery outcomes
}

\author{
Abstracted from \\ Setzer FC, Kohli MR, Shah SB, Karabucak B, Kim S. \\ Outcome of endodontic surgery: a meta-analysis of the literature - Part 2: Comparison of \\ endodontic microsurgical techniques with and without the use of higher magnification. \\ J Endod 2012; 38: 1-10. Epub 2011 Nov 21. Review. PubMed PMID: 22152611. \\ Address for correspondence: Frank C. Setzer, DMD, PhD, MS, Instructor, \\ Department of Endodontics, School of Dental Medicine, University of Pennsylvania, \\ 240 S 40th St, Philadelphia, PA 19104, USA. E-mail: fsetzer@dental.upenn.edu
}

\section{Question: Does endodontic microsurgery (EMS) have better outcomes than contemporary root-end surgery (CRS)?}

Data sources Medline, Embase and PubMed databases were searched together with hand-searches of a range of journals (Journal of Endodontics, International Endodontic Journal, Oral Surgery Oral Medicine Oral Pathology Oral Radiology and Endodontics, Journal of Oral and Maxillofacial Surgery, International Journal of Oral and Maxillofacial Surgery).

Study selection Clinical studies in several languages (English, French, German, Italian and Spanish) with a minimum follow-up of six months evaluated using clinical and radiographic examination included.

Data extraction and synthesis Assessment and data abstraction were carried out independently. Weighted pooled success rates and relative risk assessment between TRS and EMS were calculated and a meta-analysis was carried out using a random effects model. Results Fourteen studies met the inclusion criteria. Weighted pooled success rates calculated from extracted raw data showed an $88 \%$ positive outcome for CRS (95\% confidence interval [CI], 0.8455 $0.9164)$ and $94 \%$ for EMS $(95 \% \mathrm{Cl}, 0.8889-0.9816)$. This difference was statistically significant $(\mathrm{P}<.0005)$.

Relative risk ratio analysis showed that the probability of success for EMS was 1.07 times that for CRS. Seven studies provided information on the individual tooth type. The probability of success between the groups was statistically significant in favour of EMS for molars, but no significant difference was found for anteriors or premolars.

Conclusions The probability for success for EMS proved to be significantly greater than the probability for success for CRS, providing best available evidence on the influence of high-power magnification rendered by the dental operating microscope or the endoscope.

\section{Commentary}

The authors of this systematic review have called it Part 2. The first part of their study evaluated the probability of success of traditional root-end surgery (TRS), using surgical burs and amalgam for the root-end filling, vs. endodontic microsurgery (EMS). The results showed that EMS had statistically significant greater success than TRS. This second review, Part 2, was done to evaluate whether this success was due to the use of microscope or the other more innovative procedures utilised with EMS.

Successful endodontic surgery depends on many variables. Some factors that affect the outcome are the number of roots the tooth has, canal anatomy, the area size of apical pathology, access to the roots, post-op sequelae and the ability of root-end filling material to be biocompatible and remain sealed. Using EMS technique utilising the microscope, micro-instruments, ultrasonic tips and more biologically acceptable and durable root-end filling materials, gives the practitioner greater control of some of these variables. Is it the use of the microscope, or is it these other accessory tools, referred to by the authors as contemporary root-end surgery (CRS), that actually affects the outcome?

In Part 2, a search of the literature produced a total of 16 studies, including seven randomised control trials, for meta-analysis. Again, languages were limited to English, French, German, Spanish and Italian. The authors, however, strengthened this study by giving results for molars, premolars and anterior teeth. Molars were shown to have a statistically significant greater probability of success with EMS than with CRS. The success rate for premolars and anterior teeth was slightly greater for EMS than CRS, but was not statistically significant (the CI for the odds ratio included 0).

Using micro-instruments allows for good access with less bone destruction. ${ }^{1}$ Ultrasonic tips give the operator greater control and a cleaner preparation. Newer filling materials such as IRM, Super EBA and MTA allow for greater durability and biocompatibility. The microscope enables one to see the anatomy of the root- end with more clarity. One may conclude from the study that all of these factors, including the microscope, increase the probability of success significantly for molar teeth. Both premolars and anterior teeth also had an advantage in outcome with EMS, although not a statistically significant one. The field would benefit from more large-scale randomised control trials.

David Levenson

NYU College of Dentistry, New York, USA

1. Kim S, Kratchman S. Modern endodontic surgery concepts and practice: a review. J. Endod. 2006; 32: 601-623.

Evidence-Based Dentistry (2012) 13, 109. doi:10.1038/sj.ebd.6400893 